Review Article: Open Access

\title{
Nanoparticles: From Diagnosis to Therapy
}

\section{Derya İlem-Özdemir*, Evren Gündoğdu, Meliha Ekinci and Makbule Aşikoğlu}

\author{
Department of Radiopharmacy, Ege University, Izmir, Turkey \\ *Corresponding author: Derya llem-Ozdemir, Faculty of Pharmacy, Department of Radiopharmacy, Ege University, \\ Bornova, Izmir, Turkey, Tel: +90-533-446-40-26, Fax: +90-232-388-52-58, E-mail: deryailem@gmail.com
}

\begin{abstract}
Cancer is a leading cause of death, the second most common cause, exceeded only by heart disease. Still, the current clinical imaging methods and treatments are in many situations unable to provide timely detection and curative therapy. The field of drug delivery stands to be significant advances in nanotechnology and benefits of novel nanotechnology in oncology already starts. New strategies are being designed to deliver chemotherapeutic drugs or imaging agents to the tumor at higher concentrations with minimal damage to normal tissues. This review will focus on how nanoparticles are able to function as carriers for chemotherapeutic drugs to increase their therapeutic index; how can be used as imaging agents to detect and monitor cancer progression.
\end{abstract}

\section{Keywords}

Nanoparticle, Diagnosis, Therapy, Radiopharmaceutical

\section{Introduction}

Cancer is a leading cause of death, the second most common cause in the world, exceeded only by heart disease and is a complex group of diseases with many possible causes. The causes of cancer include genetic factors; lifestyle factors such as tobacco use, diet, and physical activity; certain types of infections; and environmental exposures to different types of chemicals and radiation. According to the American Cancer Society, about many Americans were expected to die in 2011 due to cancer, and that means more than 1,500 deaths per day.

There are common types of cancer treatment, such as surgery, chemotherapy, radiation therapy, and many others. Learn how they work and why they are used, and get an idea of what to expect and how they might affect you if you're getting them. Although these conventional therapies have improved patients' survival, they also have several limitations. For example, conventional cancer chemotherapy has the cancer therapeutic agents distributing nonspecifically in the human body, thus these drugs affect both cancerous and normal cells. This non-specific distribution of drugs limits the therapeutic dose within cancer cells while providing excessive toxicities to normal cells, tissues, and organs; and thereby causing several adverse side effects including hair loss, weakness, and organ function, leading to a low quality of life for cancer patients [1-7].

Chemotherapy is the use of medicines or drugs to treat cancer. The thought of having chemotherapy frightens many people. After completion of chemotherapy treatment, it is toxicity of normal cells that constrains dose and frequency both important factors in the persistence of cancer cells. Attempts are now focused on efforts to kill cancer cells by more specific targeting while sparing normal cells. To achieve these goals, the focus is the development of novel carriers for both existing and new drugs and defining better therapeutic targets relative to the molecular changes in the cancer cells, their vasculature, and the related stroma.

A tumor cell mass obtains nutrients for growth by passive diffusion until it reaches a size of about $2 \mathrm{~mm}^{3}$. To continue growth, new blood vessels are synthesized by tumors in a process known as angiogenesis; however, these vessels are abnormal with increased numbers of proliferating endothelial cells, increased vessel tortuosity, deficient pericytes and abnormalities in the basement membrane with large gaps between adjacent endothelial cells ranging between 380 and 780 nanometers (nm). Importantly, tumor vessels are abnormal and have aberrant branching blind loops and tortuosity. This results in enhanced permeability for molecule passage through the vessel wall into the interstitial surrounding tumor cells. This is in contradistinction to the tight endothelial junctions of normal vessels typically of 5 to $10 \mathrm{~nm}$ size [8-14].

Unlike normal tissues, tumor interstitial pressures are higher in the tumor center and lower in the periphery, favoring decreased drug diffusion to the center of tumors. Additionally, tumors lack well-defined lymphatic networks. Hence, drugs that gain interstitial access may have extended retention times in the tumor interstitial. This feature is termed the enhanced permeability and retention (EPR) effect and favors tumor interstitial drug accumulation [8-11,15,16]. Although the EPR effect helps to deliver chemotherapeutic agents to well-vascularized parts of the tumor, drugs may not reach the poorly vascularized regions, thereby preventing some cancer cells from receiving cytotoxic treatment [12].

To overcome these obstacles, current research has focused on developing more efficient local drug delivery or drugtargeted therapies. New strategies are being designed to deliver chemotherapeutic drugs to the tumor at higher concentrations with minimal damage to normal tissues. Since their advantages include enhancing solubility of hydrophobic drugs, prolonging circulation time, minimizing non-specific uptake, preventing undesirable offtarget and side effects, improving intracellular penetration, and allowing for specific cancer-targeting, nanoparticles (NPs) are used for cancer drug delivery, tumor therapy, and tumor follow-up using different imaging modalities to overcome these problems $[17,18]$.

Nanoscience and nanotechnology are concerned with materials in atomic, molecular and supramolecular levels (on a scale of 1-100 
$\mathrm{nm})$. Nanotechnology term is derived from "nano" which means are one billionth or $10^{-9}$ and dwarf in Greek [19-22]. Since everything is made of atoms and molecules, nanotechnology is an interdisciplinary research field, starting with materials and physics and later extending to biology and medicine $[2,21,23]$.

Nanomedicine can be defined as the monitoring, repair, construction and control of human biological systems at the molecular level, using engineered nanodevices and nanostructures [24]. The overall goal of nanomedicine is to diagnose as accurately and early as possible, to treat as effectively as possible with minimal side effects and to evaluate the efficacy of treatment noninvasively [25]

NPs may be defined as being submicronic (b1 $\mu \mathrm{m})$ colloidal systems. According to the process used for the preparation of the NPs, nanospheres or nanocapsules can be obtained. Unlike nanospheres, nanocapsules are vesicular systems in which the drug is confined to an aqueous or oily cavity surrounded by a single polymeric membrane. Thus, nanocapsules may be considered as a 'reservoir' system [26,27]

NPs can be prepared with high flexibility for the purpose of obtaining various functionalities within the NP matrix and its surface. NP has high surface area. This property is making the NPs surface and its internal space available for encapsulating with a high amount of drugs/contrast agents or targeting moieties. Due to the engineer ability, as well as the non-toxic nature of its matrix, NPs have shown many advantages when used as drug delivery systems and contrast agents and they can be especially suitable for molecular targeted approaches [28-31].

The field of drug delivery stands to be significant advances in nanotechnology and benefits of novel nanotechnology in oncology already starts. The National Cancer Institute (NCI) has identified nanotechnology as having the potential to make paradigm-changing impacts on the detection, treatment, and prevention of cancer $[28,32,33]$

This review will focus on how NPs are able to function as carriers for chemotherapeutic drugs to increase their therapeutic index; how can be used as imaging agents to detect and monitor cancer progression.

\section{NPs in Clinical Use}

NPs have been widely used in clinical practice. Over the last two decades, a large number of NP delivery systems have been developed for clinical use, including organic and inorganic materials. Many polymer-drug conjugates, liposomal systems, micelles systems, NPs are examples of formulations used in the clinic. In addition, NPs are used in the development of preclinical stages [34]. Many interesting in vitro and animal studies have been reported and some NP-based products are already in clinical use [35].

NPs offer advantages for clinical use mainly in two areas:

i. Targeted therapeutics: delivering drugs where they are needed.

ii. Tissue engineering: building new tissues to replace defective valves, damaged muscle, blood vessels, etc. [36].

The most important clinical applications of nanotechnology are in area of pharmaceutical development and pharmaceutical NPs have gained great importance for the clinical use. In pharmaceutical technology and biomedicine, NPs are typically defined as particles with diameter from 1 to $100 \mathrm{~nm}$ and have been exploited for both diagnostic and therapeutic purposes. The ideal size of NPs used as drug delivery systems ranges from 10 to $100 \mathrm{~nm}$. They can be classified into several categories, according to their structure (i.e. NPs, nanospheres, nanocapsules, nanotubes and colloidal carriers such as liposomes or dendrimers), physicochemical properties (i.e., $\mathrm{pH}$ sensitive, magnetic, stealth NPs) and the materials used for its synthesis (i.e. natural, synthetic, hybrid, or gold NPs (AuNPs)). NPs can achieve controlled drug release, targeting and increase the effectiveness or bioavailability of many therapeutic agents [36-38].

\section{NPs in Cancer Therapy}

Nanotechnology is not only a new tool to fight cancer, it is also a novel research field in therapy development, from which new generations of diagnostics, imaging and drug treatments have emerged. Over $20 \%$ of the therapeutic nanoparticulate systems already in clinics were developed for anticancer applications. Using targeted NPs to deliver chemotherapeutic agents in cancer therapy offers many advantages to improve drug/gene delivery and to overcome many problems associated with conventional chemotherapy $[6,7,39$ 44].

NPs can enhance the intracellular concentration of drugs in cancer cells by using both passive and active targeting strategies, while avoiding toxicity in normal cells. Furthermore, when NPs bind to specific receptors and then enter the cell, they are usually enveloped by endosomes via receptor-mediated endocytosis, thereby by passing the recognition of P-glycoprotein, one of the main drug resistance mechanisms $[30,45,46]$.

Although NPs offer many advantages as drug delivery systems, there are still many limitations to be solved. For instance, NPs might change the stability, solubility, and pharmacokinetic properties of the carried drugs. However, these aspects have not been extensively investigated. The shelf life, aggregation, leakage and toxicity of materials used to make NPs are other limitations for their use [47].

Various types of NPs have been studied as drug delivery vehicles for anticancer drugs, which includes poly (lactic-co-glycolic acid) (PLGA) and its derivatives, chitosan, dendrimer, solid lipid NP (SLNP), low density lipoprotein (LDL), polysorbate 80-coated poly (butyl cyanoacrylate) NP, layered double hydroxides (LDH) and mesoporous silica nanoparticles (MSN) [48-57]. Despite extensive research and development, only a few drug delivery NPs are approved by 'Food and Drug Administration (FDA)' and available for cancer treatment. Liposomal anticancer drugs were the first to be approved by FDA for cancer therapy [8].

In cancer treatments, NPs can rely on the enhanced permeability and retention effect caused by leaky tumor vasculatures for better drug accumulation at the tumor sites. These benefits have made that therapeutic NPs are promising candidate to replace traditional chemotherapy, where intravenous injection of toxic agents poses a serious threat to healthy tissues and results in dose-limiting side effects. Currently, several NP-based chemotherapeutics have emerged on the market, while many of them are undergoing various stages of clinical or preclinical development. Notable examples of chemotherapeutic NPs include Doxil $^{{ }^{\bullet}}$ (a $\sim 100 \mathrm{~nm}$ liposomal formulation of doxorubicin) and Abraxane (a $\sim 130 \mathrm{~nm}$ paclitaxelbound protein particle), both of which are routinely administered as first-line treatments in various cancer types [58-62].

The early stage cancer is treatable, in general, and thus the prognosis could be good. Cancer diagnosis is thus an important and practical way to improve the cure rate. The current practice of cancer diagnosis prefers invasive tissue biopsies to confirm the diagnosis of cancer as this can also provide information about its histological type, classification, grade, and potential aggressiveness. It is useful to identify a fully developed cancer, but not so efficient to detect the pre-malignant or early lesions (intermediate stages) amenable to resection and cure [58-61].

\section{NPs in Diagnosis}

Conventional imaging by using plain radiographs, ultrasound, computed tomography (CT), and magnetic resonance imaging (MRI) has been common in both cancer screening and follow-up. However, all these modalities rely on detecting cancer once it becomes a visible physical entity, at around $1 \mathrm{~cm}^{3}$ at which point the tumor mass will already contain approximately 1 billion cancer cells. Over the past decade, there has been a paradigm shift from anatomical imaging, which detects macroscopic/gross pathology, to molecular imaging, which has the potential to detect cancer much earlier at the molecular 
level, long before phenotypic changes occur. Molecular imaging allows the genetic changes involved in oncogenesis to be characterized in vivo, thereby predicting the type of molecular therapy that will prove most beneficial for the patient (i.e. personalized medicine). It also allows the repeated noninvasive monitoring of the disease for response, progression, and transformation following therapy or recurrence $[12,63]$.

Molecular imaging is one of the main current focuses of research in medical diagnostics, which can facilitate early diagnosis, identify the stage of disease, provide fundamental information on pathological processes, and can be applied to follow the efficacy of therapy [64]. NPs probes have the potential for molecular imaging from its current anatomy based level to the molecular level, can travel through the human body in the blood and lymphatic vessels. They can identify the desired target by specific biological interactions, such as antibody antigen, nucleic acid hybridization and gene expression [65]. For molecular imaging four classes of NPs are primary interest: magnetic NPs, magneto fluorescent NPs, florescent NPs and isotope tagged NPs. Nanotechnology-enabled molecular imaging has been made in all imaging modalities including optical, ultrasound (US), CT, MRI and nuclear imaging. Each imaging modality has its own unique advantages and disadvantages. Ultimately the choice will be determined by the specific molecular process that is being targeted [66-73].

Magnetic NPs were used directly or dispersed in the polymeric matrix as cores. The use of magnetic NPs is having a great impact in bioassays for separation and pre concentration. Recently, magnetic micro/nano-particles have been widely used as signal reporters to detect various biomolecules, and to facilitate location of cancerous cells [74-76]. They have been used chiefly in the hyperthermia treatment of cancer and, in some cases, for magnetic field assisted targeting of NPs. For diagnostic purposes, they were used in MRI as contrast-enhancing agents for the purpose of cancer diagnosis, targeted molecular imaging, hypoperfusion region visualization, cell labeling in T cell-based therapy, and for detection of angiogenesis, apoptosis and gene expression [66,77-85].

Magnetic and fluorescent inorganic NPs are of particular importance due to their broad range of potential applications. It is expected that the combination of magnetic and fluorescent properties in one nanocomposite would enable the engineering of unique multifunctional nanoscale devices, which could be manipulated using external magnetic fields. First of all, multi-modal magnetic-fluorescent assays would be very beneficial for in vitro- and in vivobioimaging applications such as MRI and fluorescence microscopy. Second of all, these nanocomposites can be utilised as agents in nanomedicine. Fluorescent-magnetic nanocomposites can also serve as an all-in-one diagnostic and therapeutic tool, which could be used, for example, to visualise and simultaneously treat various diseases [86].

The application of nanotechnology to cancer diagnosis holds tremendous promise in enhancing the sensitivity and versatility of fluorescence-based methods of detection. In particular, there are several structure-defining traits of NPs that enable the development of novel cancer detection assays: size, shape, high surface area, and unique optical properties.In particular, the optical properties most relevant in the design of fluorescence-based biosensors for cancer diagnostics, the intensity and stability of fluorescence emission as well as the effectiveness of fluorescence quenching in "off-on" probes, determine, in part, the sensitivity and dynamic range of a particular assay. The four main NP types used in the design of probes for the detection of cancer biomarkers, cells, and tissues through fluorescence are [1] QDs, which exhibit tunable absorption and high quantum yields; [2] pee-dots (PDs), which have high fluorescence quantum yields and show no cytotoxicity; [3] up-convertion NPs (UCNPs), which exhibit anti-Stokes shifts and are excitable in the tissue-transparent near infrared (NIR) window; [4] AuNPs, which are excellent fluorescence quenchers and are thus commonly used in "off-on" probes; and [5] fluorophore-encapsulating polymeric or mesoporous silica NPs, which are useful in theranostic applications
Table 1: Radionuclides for diagnosis and therapy.

\begin{tabular}{|c|c|c|c|c|}
\hline Radionuclide & Half-life & Emission type & Emax & References \\
\hline \multicolumn{5}{|c|}{ DIAGNOSIS } \\
\hline${ }^{99 \mathrm{~m}} \mathrm{Tc}$ & 6.0 hour & $\gamma$ & $141 \mathrm{KeV}$ & {$[94]$} \\
\hline${ }^{18} \mathrm{~F}$ & $109.8 \mathrm{~min}$ & $\beta^{+}$ & $634 \mathrm{KeV}$ & [95] \\
\hline${ }^{64} \mathrm{Cu}$ & 12.7 hour & $\beta^{-}, \beta^{+}$ & $579,653 \mathrm{KeV}$ & [96] \\
\hline 123 | & 13.2 hour & Auger $e^{-}, \gamma$ & $159 \mathrm{KeV}$ & [92] \\
\hline${ }^{124} \mid$ & 4.18 days & $\beta^{+}, \gamma$ & $820,1543,2146 \mathrm{KeV}$ & {$[97]$} \\
\hline 131 I & 8.0 days & $\gamma, \beta$ & $284,364,637 \mathrm{KeV}$ & [92] \\
\hline${ }^{111}$ In & 2.8 days & $\gamma$, Auger $\mathrm{e}^{-}$ & $172,245 \mathrm{KeV}$ & [98] \\
\hline${ }^{67} \mathrm{Ga}$ & 3.3 days & $\gamma$ & $93,185,296 \mathrm{KeV}$ & [98] \\
\hline${ }^{68} \mathrm{Ga}$ & $67.7 \mathrm{~min}$ & $\beta^{+}$ & $770,1890 \mathrm{KeV}$ & [98] \\
\hline \multicolumn{5}{|c|}{ THERAPY } \\
\hline 131 I & 8.0 days & $\gamma, \beta$ & $0.28,0.36,0.64 \mathrm{MeV}$ & [97] \\
\hline${ }^{67} \mathrm{Cu}$ & 2.6 days & $\beta$ & $0.19 \mathrm{MeV}$ & [97] \\
\hline${ }^{186} \mathrm{Re}$ & 89.2 hour & $\beta, \gamma$ & $1.07 \mathrm{MeV}$ & [92] \\
\hline${ }^{188} \mathrm{Re}$ & 17.0 hour & $\beta, \gamma$ & 2.12 MeV & [92] \\
\hline${ }^{177} \mathrm{Lu}$ & 161.0 hour & $\beta$ & $0.49 \mathrm{MeV}$ & [97] \\
\hline${ }^{90} \mathbf{Y}$ & 64.1 hour & $\beta$ & $2.28 \mathrm{MeV}$ & [97] \\
\hline${ }^{225} \mathrm{Ac}$ & 10.0 days & $\alpha$ & $5.83,5.79,5.73 \mathrm{MeV}$ & [92] \\
\hline${ }^{211} \mathrm{At}$ & 7.2 hour & $\alpha$ & $5.87 \mathrm{MeV}$ & [92] \\
\hline 111 In & 67.0 hour & Auger $e^{-}, \gamma$ & $0.42 \mathrm{MeV}$ & [97] \\
\hline
\end{tabular}

that simultaneously deliver chemotherapeutics or photosensitizing agents to image and treat cancer cells $[87,88]$.

Due to their enormous flexibility, and versatility the radiolabeled NPs have shown their potential in the diagnosis and therapy. As the matter of fact, these radiolabeled imaging agents enable the visualization of the cellular function and the follow-up of the molecular process in living organisms. Many different kinds of particles have been loaded or labeled with various radionuclides and used in nuclear medicine for diagnostic, therapeutic and investigational purposes. Nuclear medicine is a branch of medical imaging that uses small amounts of radioisotopes to diagnose a wide variety of diseases and to treat many disorders. Nuclear medicine imaging differs from other radiological imaging techniques in that radiotracers are administrated to the patients and the emitted radiation is images relate to the function of an organ system or metabolic pathway. Different imaging studies include dynamic or static imaging and in vivo function tests perform in nuclear medicine. The acquired information is useful for both diagnostic purposes, such as detection of functional abnormalities or early identification of tumors and therapy planning and follow-up. Choosing the radionuclide compounds play a major role for medicine and biology (Table 1) [89-93].

Various approaches are used for labeling radionuclides are the surface labeling of the NP after encapsulation or encapsulating a radiolabeling NP. However, the NPs conjugated with bifunctional chelators and targeting ligands are particularly useful because their higher surface area which allows a higher number of targeting residues and radionuclides per particle.

\section{Conclusion}

NPs designed in various ways have been widely investigated for application to cancer therapy and diagnosis. This is common to chemotherapy, gene therapy and modalities including optical, ultrasound, CT, MRI and nuclear imaging. The imaging contrast and the therapeutic efficiency of drugs have improved significantly due to the high payload of drug/contrast agents per NP and the ability for active/passive targeting. NP-based imaging and therapy demonstrated reduced side effects, too. Different NPs have been elaborated for diagnostic applications with promising results. Since nuclear medicine is the most sensitive clinical imaging techniques with between nanomole/kilogram and picomole/kilogram sensitivity, imaging technique with radioactive NPs is becoming increasingly important. Significant advantages of radioactive imaging, other than sensitivity are, they are quantitative and there is no tissue penetration limit. With these promising advantageous of NP based imaging, it is important to understand to design and apply NPs for diagnosis. 


\section{References}

1. Yigit MV, Moore A, Medarova Z (2012) Magnetic nanoparticles for cancer diagnosis and therapy. Pharm Res 29: 1180-1188.

2. Pautler M, Brenner S (2010) Nanomedicine: promises and challenges for the future of public health. Int J Nanomedicine 5: 803-809.

3. Kievit FM, Zhang M (2011) Surface engineering of iron oxide nanoparticles for targeted cancer therapy. Acc Chem Res 44: 853-862.

4. Nguyen KT (2011) Targeted nanoparticles for cancer therapy: promises and challenges. J Nanomedic Nanotechnol 2: 5.

5. Subbiah R, Veerapandian M, Yun KS (2010) Nanoparticles: functionalization and multifunctional applications in biomedical sciences. Curr Med Chem 17 4559-4577.

6. Yoo JW, Chambers E, Mitragotri S (2010) Factors that control the circulation time of nanoparticles in blood: challenges, solutions and future prospects. Curr Pharm Des 16: 2298-2307.

7. Koo H, Huh MS, Sun IC, Yuk SH, Choi K, et al. (2011) In vivo targeted delivery of nanoparticles for theranosis. Acc Chem Res 44: 1018-1028.

8. Haley B, Frenkel E (2008) Nanoparticles for drug delivery in cancer treatment. Urol Oncol 26: 57-64

9. Jones A, Harris AL (1998) New developments in angiogenesis: a majo mechanism for tumor growth and target for therapy. Cancer J Sci Am 4: 209 217

10. Baban DF, Seymour LW (1998) Control of tumour vascular permeability. Adv Drug Deliv Rev 34: 109-119.

11. Hobbs SK, Monsky WL, Yuan F, Roberts WG, Griffith L, et al. (1998) Regulation of transport pathways in tumor vessels: role of tumor type and microenvironment. Proc Natl Acad Sci U S A 95: 4607-4612.

12. Thakor AS, Gambhir SS (2013) Nanooncology: the future of cancer diagnosis and therapy. CA Cancer J Clin 63: 395-418.

13. Kanapathipillai M, Brock A, Ingber DE (2014) Nanoparticle targeting of anti-cancer drugs that alter intracellular signaling or influence the tumor microenvironment. Adv Drug Deliv Rev 79-80: 107-18.

14. Shu Y, Pi F, Sharma A, Rajabi M, Haque F, et al. (2014) Stable RNA nanoparticles as potential new generation drugs for cancer therapy. Adv Drug Deliv Rev 66: 74-89.

15. Jain RK (2001) Delivery of molecular and cellular medicine to solid tumors Adv Drug Deliv Rev 46: 149-168.

16. Maeda H, Wu J, Sawa T, Matsumura Y, Hori K (2000) Tumor vascular permeability and the EPR effect in macromolecular therapeutics: a review. $J$ Control Release 65: 271-284.

17. Liu Y, Miyoshi H, Nakamura M (2007) Nanomedicine for drug delivery and imaging: a promising avenue for cancer therapy and diagnosis using targeted functional nanoparticles. Int J Cancer 120: 2527-2537.

18. Torchilin VP (2007) Targeted pharmaceutical nanocarriers for cancer therapy and imaging. AAPS J 9: E128-147.

19. Sahoo SK, Labhasetwar $\vee(2003)$ Nanotech approaches to drug delivery and imaging. Drug Discov Today 8: 1112-1120.

20. Balzani V (2005) Nanoscience and nanotechnology: a personal view of a chemist. Small 1: 278-283.

21. Roco MC (2003) Nanotechnology: convergence with modern biology and medicine. Curr Opin Biotechnol 14: 337-346.

22. Linazasoro G; Nanotechnologies for Neurodegenerative Diseases Study Group of the Basque Country (NANEDIS) (2008) Potential applications of nanotechnologies to Parkinson's disease therapy. Parkinsonism Relat Disord 14: 383-392.

23. Bassecoulard E, Lelu A, Zitt M (2007) Mapping nanosciences by citation flows: A preliminary analysis. Scientometrics $70: 859-880$

24. Sahoo SK, Parveen S, Panda JJ (2007) The present and future of nanotechnology in human health care. Nanomedicine 3: 20-31.

25. Caruthers SD, Wickline SA, Lanza GM (2007) Nanotechnological applications in medicine. Curr Opin Biotechnol 18: 26-30.

26. Couvreur P, Couarraze G, Devissaguet J-P, Puisieux F (1996) Nanoparticles: preparation and characterization, In: Benita S, Microencapsulation: Methods and Industrial Application. Marcel Dekker, New York, 183-211.

27. Brigger I, Dubernet C, Couvreur P (2002) Nanoparticles in cancer therapy and diagnosis. Adv Drug Deliv Rev 54: 631-651.

28. Koo Lee YE, Orringer DA, Kopelman R (2010) Nanoparticles for cance diagnosis and therapy. In: Broz P, Polymer-based Nanostructures Medical Applications. Royal Society of Chemistry, Thomas Graham House, Science Park, Milton Road, Cambridge, UK, 333-353.
29. Porkka K, Laakkonen P, Hoffman JA, Bernasconi M, Ruoslahti E (2002) A fragment of the HMGN2 protein homes to the nuclei of tumor cells and tumor endothelial cells in vivo. Proc Natl Acad Sci U S A 99: 7444-7449.

30. Allen TM (2002) Ligand-targeted therapeutics in anticancer therapy. Nat Rev Cancer 2: 750-763.

31. Couvreur P, Vauthier $C$ (2006) Nanotechnology: intelligent design to treat complex disease. Pharm Res 23: 1417-1450.

32. Alexis $F$, Basto $P$, Levy-Nissenbaum E, Radovic-Moreno AF, Zhang L, et al. (2008) HER-2-targeted nanoparticle-affibody bioconjugates for cancer therapy. ChemMedChem 3: 1839-1843.

33. Alexis F, Pridgen E, Molnar LK, Farokhzad OC (2008) Factors affecting the clearance and biodistribution of polymeric nanoparticles. Mol Pharm 5: 505515.

34. Alexis F, Pridgen EM, Langer R, Farokhzad OC (2010) Nanoparticle technologies for cancer therapy. Handb Exp Pharmacol : 55-86

35. Skotland T, Iversen TG, Sandvig K (2014) Development of nanoparticles for clinical use. Nanomedicine (Lond) 9: 1295-1299.

36. Arayne MS, Sultana N, Qureshi F (2007) Review: nanoparticles in delivery of cardiovascular drugs. Pak J Pharm Sci 20: 340-348.

37. Goldberg M, Langer R, Jia X (2007) Nanostructured materials for applications in drug delivery and tissue engineering. J Biomater Sci Polym Ed 18: 241-268.

38. Gündogdu E, Senyigit Z, llem Ozdemir D (2014) Nanomedicine for diagnosis and treatment of cardiovascular disease: Current status and future perspective. Cardiovascular Disease I: 187-200.

39. Ali I, Rahis-Uddin, Salim K, Rather MA, Wani WA, et al. (2011) Advances in nano drugs for cancer chemotherapy. Curr Cancer Drug Targets 11: 135-146.

40. Heidel JD, Davis ME (2011) Clinical developments in nanotechnology for cancer therapy. Pharm Res 28: 187-199.

41. Davis ME, Chen ZG, Shin DM (2008) Nanoparticle therapeutics: an emerging treatment modality for cancer. Nat Rev Drug Discov 7: 771-782.

42. Heath JR, Davis ME (2008) Nanotechnology and cancer. Annu Rev Med 59 251-265.

43. Peer D, Karp JM, Hong S, Farokhzad OC, Margalit R, et al. (2007) Nanocarriers as an emerging platform for cancer therapy. Nat Nanotechnol 2: $751-760$

44. Service RF (2005) Materials and biology. Nanotechnology takes aim at cancer. Science 310: 1132-1134.

45. Maeda $H$ (2001) The enhanced permeability and retention (EPR) effect in tumor vasculature: the key role of tumor-selective macromolecular drug targeting. Adv Enzyme Regul 41: 189-207.

46. Mulvana H, Reboud J, de Scrilli M, Berry C (2015) Uptake and cellula recovery mechanisms in microbubble-enhanced ultrasound delivery of nanoparticles for cancer therapy. IEEE International Ultrasonics Symposium Proceedings.

47. Cho K, Wang X, Nie S, Chen ZG, Shin DM (2008) Therapeutic nanoparticles for drug delivery in cancer. Clin Cancer Res 14: 1310-1316.

48. Farokhzad OC, Cheng J, Teply BA, Sherifi I, Jon S, et al. (2006) Targeted nanoparticle-aptamer bioconjugates for cancer chemotherapy in vivo. Proc Natl Acad Sci U S A 103: 6315-6320.

49. Dong Y, Feng SS (2007) Poly(D,L-lactide-co-glycolide) (PLGA) nanoparticles prepared by high pressure homogenization for paclitaxel chemotherapy. Int J Pharm 342: 208-214.

50. Hu CM, Zhang L (2009) Therapeutic nanoparticles to combat cancer drug resistance. Curr Drug Metab 10: 836-841.

51. Kukowska-Latallo JF, Candido KA, Cao Z, Nigavekar SS, Majoros IJ, et al. (2005) Nanoparticle targeting of anticancer drug improves therapeutic response in animal model of human epithelial cancer. Cancer Res 65: 5317-5324.

52. Nagayama S, Ogawara K, Fukuoka Y, Higaki K, Kimura T (2007) Timedependent changes in opsonin amount associated on nanoparticles alter their hepatic uptake characteristics. Int J Pharm 342: 215-221.

53. Nikanjam M, Gibbs AR, Hunt CA, Budinger TF, Forte TM (2007) Synthetic nano-LDL with paclitaxel oleate as a targeted drug delivery vehicle for glioblastoma multiforme. J Control Release 124: 163-171.

54. De Juan BS, Von Briesen H, Gelperina SE, Kreuter J (2006) Cytotoxicity of doxorubicin bound to poly(butyl cyanoacrylate) nanoparticles in rat glioma cell lines using different assays. J Drug Target 14: 614-622.

55. Steiniger SC, Kreuter J, Khalansky AS, Skidan IN, Bobruskin Al, et al. (2004) Chemotherapy of glioblastoma in rats using doxorubicin-loaded nanoparticles. Int J Cancer 109: 759-767.

56. Slowing II, Vivero-Escoto JL, Wu CW, Lin VS (2008) Mesoporous silica nanoparticles as controlled release drug delivery and gene transfection carriers. Adv Drug Deliv Rev 60: 1278-1288. 
57. Li F, Duan X (2006) Applications of layered double hydroxides. Struct Bond 119: $193-223$

58. Matsumura Y, Maeda H(1986)A new concept for macromolecular therapeutics in cancer chemotherapy: mechanism of tumoritropic accumulation of proteins and the antitumor agent smancs. Cancer Res 46: 6387-6392.

59. Brannon-Peppas L, Blanchette JO (2004) Nanoparticle and targeted systems for cancer therapy. Adv Drug Deliv Rev 56: 1649-1659.

60. Northfelt DW, Dezube BJ, Thommes JA, Miller BJ, Fischl MA, et al. (1998) PEGylated-liposomal doxorubicin versus doxorubicin, bleomycin and vincristine in the treatment of AIDS-related Kaposi's sarcoma: results of a randomized phase III clinical trial. J Clin Oncol 16: 2445-2451.

61. Harries M, Ellis $P$, Harper $P$ (2005) Nanoparticle albumin-bound paclitaxel for metastatic breast cancer. J Clin Oncol 23: 7768-7771.

62. Schleich N, Po C, Jacobs D, Ucakar B, Gallez B, et al. (2014) Comparison of active, passive and magnetic targeting to tumors of multifunctional paclitaxel/ SPIO-loaded nanoparticles for tumor imaging and therapy. J Control Release 194: 82-91.

63. Siegmund KD, Marjoram P, Woo YJ, Tavaré S, Shibata D (2009) Inferring clonal expansion and cancer stem cell dynamics from DNA methylation patterns in colorectal cancers. Proc Natl Acad Sci U S A 106: 4828-4833.

64. Cormode DP, Skajaa T, Fayad ZA, Mulder WJ (2009) Nanotechnology in medical imaging: probe design and applications. Arterioscler Thromb Vasc Biol 29: 992-1000.

65. Jun YW, Lee JH, Cheon J (2008) Chemical design of nanoparticle probes for high-performance magnetic resonance imaging. Angew Chem Int Ed Engl 47: $5122-5135$

66. Moffat BA, Reddy GR, McConville P, Hall DE, Chenevert TL, et al. (2003) A novel polyacrylamide magnetic nanoparticle contrast agent for molecular imaging using MRI. Mol Imaging 2: 324-332.

67. Bremer C, Ntziachristos V, Weissleder R (2003) Optical-based molecula imaging: contrast agents and potential medical applications. Eur Radiol 13: 231-243.

68. Lanza GM, Wickline SA (2003) Targeted ultrasonic contrast agents for molecular imaging and therapy. Curr Probl Cardiol 28: 625-653.

69. Winter PM, Shukla HP, Caruthers SD, Scott MJ, Fuhrhop RW, et al. (2005) Molecular imaging of human thrombus with computed tomography. Acad Radiol 12 Suppl 1: S9-13.

70. Britz-Cunningham SH, Adelstein SJ (2003) Molecular targeting with radionuclides: state of the science. J Nucl Med 44: 1945-1961.

71. Caruthers SD, Winter PM, Wickline SA, Lanza GM (2006) Targeted magnetic resonance imaging contrast agents. Methods Mol Med 124: 387-400.

72. Jaffer FA, Libby P, Weissleder R (2007) Molecular imaging of cardiovascular disease. Circulation 116: 1052-1061.

73. Groneberg DA, Giersig M, Welte T, Pison U (2006) Nanoparticle-based diagnosis and therapy. Curr Drug Targets 7: 643-648.

74. Hens AG, Romero JMF, Caballos MPA (2008) Nanostructures as analytical tools in bioassays. Trends in Anal Chem 27: 394-406.

75. Munir A, Wang J, Li Z, Zhou HS (2010) Numerical analysis of a magnetic nanoparticle-enhanced microfluidic surface-based bioassay. Microfluid Nanofluid 8: 641-652.

76. Lim YT, Cho MY, Choi BS, Lee JM, Chung BH (2008) Paramagnetic gold nanostructures for dual modal bioimaging and phototherapy of cancer cells. Chem Commun (Camb) : 4930-4932.

77. Ito A, Shinkai M, Honda H, Kobayashi T (2005) Medical application of functionalized magnetic nanoparticles. J Biosci Bioeng 100: 1-11.

78. Kondo A, Ohnishi N (2003) In: M. Ueda, Frontier of Nano-biotechnology, CMC, Tokyo, 66-70.
79. Bjørnerud A, Johansson LO, Ahlström HK (2001) Pre-clinical results with Clariscan (NC100150 Injection); experience from different disease models. MAGMA 12: 99-103.

80. Kircher MF, Allport JR, Graves EE, Love V, Josephson L, et al. (2003) In vivo high resolution three-dimensional imaging of antigen-specific cytotoxic T-lymphocyte trafficking to tumors. Cancer Res 63: 6838-6846.

81. Josephson L, Tung CH, Moore A, Weissleder R (1999) High-efficiency intracellular magnetic labeling with novel superparamagnetic-Tat peptide conjugates. Bioconjug Chem 10: 186-191.

82. Zhao M, Kircher MF, Josephson L, Weissleder R (2002) Differential conjugation of tat peptide to superparamagnetic nanoparticles and its effect on cellular uptake. Bioconjug Chem 13: 840-844.

83. Schellenberger EA, Bogdanov A Jr, Högemann D, Tait J, Weissleder R, et al (2002) Annexin V-CLIO: a nanoparticle for detecting apoptosis by MRI. Mol Imaging 1: 102-107.

84. Zhang Y, Kohler N, Zhang M (2002) Surface modification of superparamagnetic magnetite nanoparticles and their intracellular uptake. Biomaterials 23: 15531561.

85. Högemann D, Josephson L, Weissleder R, Basilion JP (2000) Improvement of MRI probes to allow efficient detection of gene expression. Bioconjug Chem 11: 941-946.

86. Corr SA, Rakovich YP, Gun'ko YK (2008) Multifunctional magnetic-fluorescent nanocomposites for biomedical applications. Nanoscale Research Letters 3: 87-104.

87. Chinen AB, Guan CM, Ferrer JR, Barnaby SN, Merkel TJ, et al. (2015) Nanoparticle Probes for the Detection of Cancer Biomarkers, Cells, and Tissues by Fluorescence. Chem Rev 115: 10530-10574.

88. Satterlee AB, Yuan H, Huang $L$ (2015) A radio-theranostic nanoparticle with high specific drug loading for cancer therapy and imaging. J Control Release 217: $170-182$.

89. Häfeli U (2002) Radioactive microspheres for medical applications. In: Cuyper MD, Bulte JWM, Physics and Chemistry Basis of Biotechnology. Springer, Netherlands, 7: 213-248.

90. Mody VV, Siwale R (2011) Application of nanoparticles in diagnostic imaging via ultrasonography. IJMU 6: 8-15.

91. Ruth TJ (2009) The uses of radiotracers in the life sciences. Rep Prog Phys 72: 1-23.

92. Hamoudeh M, Kamleh MA, Diab R, Fessi H (2008) Radionuclides delivery systems for nuclear imaging and radiotherapy of cancer. Adv Drug Deliv Rev 60: $1329-1346$

93. Buldinger TF, Vanbroclin HF (2000) Positron-Emission Tomography (PET). In: Bronzino JD, CRS Pres LLC.

94. Liu S, Edwards DS (1999) 99mTc-Labeled Small Peptides as Diagnostic Radiopharmaceuticals. Chem Rev 99: 2235-2268.

95. Miller PW, Long NJ, Vilar R, Gee AD (2008) Synthesis of 11C, 18F, 15O, and $13 \mathrm{~N}$ radiolabels for positron emission tomography. Angew Chem Int Ed Engl 47: 8998-9033.

96. Shokeen M, Anderson CJ (2009) Molecular imaging of cancer with copper-64 radiopharmaceuticals and positron emission tomography (PET). Acc Chem Res 42: 832-841.

97. Holland JP, Williamson MJ, Lewis JS (2010) Unconventional nuclides for radiopharmaceuticals. Mol Imaging 9: 1-20.

98. Wadas TJ, Wong EH, Weisman GR, Anderson CJ (2010) Coordinating radiometals of copper, gallium, indium, yttrium, and zirconium for PET and SPECT imaging of disease. Chem Rev 110: 2858-2902. 